\title{
Rapid detection of Salmonella based on loop-mediated isothermal amplification
}

\author{
Hongling Ou ${ }^{1,2}$, Yan Wang ${ }^{2}$, Jiayi Gao ${ }^{3}$, Jing Bai ${ }^{2}$, Qiaoyun Zhang ${ }^{2}$, Lei Shi ${ }^{2}$, Xinru Wang ${ }^{2}$, \\ Chengbin Wang ${ }^{1,4}$
}

${ }^{1}$ Medical School of Chinese PLA, Beijing, China; ${ }^{2}$ Department of Clinical Laboratory, PLA Rocket Characteristic Medical Center, Beijing, China; ${ }^{3}$ Clinical Laboratory Teaching and Research Department, School of Medical Laboratory, North Medical University, Zhangjiakou, China; ${ }^{4}$ Department of Clinical Laboratory Medicine, the First Medical Center, Chinese PLA General Hospital, Beijing, China

Contributions: (I) Conception and design: H Ou, X Wang, C Wang; (II) Administrative support: C Wang; (III) Provision of study materials or patients: Y Wang, Q Zhang; (IV) Collection and assembly of data: J Gao, L Shi; (V) Data analysis and interpretation: J Bai, H Ou; (VI) Manuscript writing: All authors; (VII) Final approval of manuscript: All authors.

Correspondence to: Chengbin Wang. Medical School of Chinese PLA, Beijing, China; Department of Clinical Laboratory Medicine, the First Medical Center, Chinese PLA General Hospital, Beijing 100029, China. Email: wangcb301@126.com; Xinru Wang. Department of Clinical Laboratory, PLA Rocket Characteristic Medical Center, Beijing 100088, China. Email: wangxinru@126.com.

Background Salmonella enterica is a zoonotic pathogen of substantial concern to human and animal health and is a leading cause of morbidity and mortality in people worldwide. Loop-mediated isothermal amplification (LAMP) technology is a new type of nucleic acid amplification technology, which has the characteristics of high specificity, high sensitivity, simple operation, convenience, and low cost. This study aims to establish a rapid detection method for Salmonella based on LAMP technology.

Methods: Primers were designed for Salmonella's specific conservative invA gene. Through primer screening and optimization of reaction conditions, and a LAMP method for detecting Salmonella with realtime fluorescence and visual observation results was established. The sensitivity and specificity of the method were assessed, and the accuracy was evaluated through the testing of Salmonella-contaminated and noncontaminated clinical samples.

Results: The optimal reaction temperature of LAMP was $60-65^{\circ} \mathrm{C}$, and the optimal reaction time was 25-30 minutes. The detection limits of real-time fluorescence and visual observation were both $1.4 \mathrm{pg} / \mu \mathrm{L}$. There was no cross-reactivity observed with 22 non-Salmonella species, and the specificity was $100 \%$. Additionally, 30 samples contaminated with Salmonella, 30 samples not contaminated with Salmonella, and 8 clinical samples identified as positive by bacterial culture and microbial mass spectrometry were tested. The positive coincidence rate of the detection system was $97.4 \%$ by real-time fluorescence and $89.5 \%$ by visual observation, the negative coincidence rate was $100 \%$, and the total coincidence rate was $98.5 \%$ and $94.1 \%$, respectively.

Conclusions: In the scene of infection, primary hospital, disaster area treatment and other scenarios, the conditions of environment, equipment and personnel was limited, therefore, the established real-time fluorescence and visual lamp method can provide a powerful means for the rapid detection of Salmonella.

Keywords: Loop-mediated isothermal amplification (LAMP); Salmonella; rapid detection

Submitted Apr 26, 2021. Accepted for publication Jun 11, 2021.

doi: 10.21037/apm-21-1387

View this article at: https://dx.doi.org/10.21037/apm-21-1387 


\section{Introduction}

There are a wide range of hosts for Salmonella enterica in the process of natural transmission, infection with which can be considerably harmful to human health. Humans can be infected by most serotypes of Salmonella, with Salmonella enteritidis and Salmonella typhimurium accounting for $70 \%$ of total cases (1). The traditional method of bacteria isolation and biochemical identification is considered as the gold standard of Salmonella detection, but it is timeconsuming. The test results can be obtained at least 3-5 days after the entire process has been completed. Molecular biological detection methods for pathogens have been widely studied and applied (2-6); however, these require professional expertise and expensive experimental equipment. This is not conducive to the rapid detection of Salmonella or the identification of infections when there is a shortage of personnel and equipment, or at primary medical institutions, incident scenes, and disaster areas.

Loop-mediated isothermal amplification (LAMP) technology is a new type of nucleic acid amplification technology that can amplify specific target sequences to $10^{9}$ levels within 1 hour at a constant temperature of 60-65 ${ }^{\circ} \mathrm{C}$. Compared with colloidal gold method, bacteria incubation, ordinary polymerase chain reactions (PCR) and other methods, it has the advantages of high specificity and sensitivity, simple operation, convenience, low cost $(7,8)$, among others. Without any special laboratory equipment, high-throughput rapid detection can be achieved onsite with the new amplification method as long as the appropriate primers are designed. Some studies have been conducted to monitor food infection. Simulating clinical samples and detection (9), In this study, LAMP primers were designed with Salmonella-specific conservative fragments, and a LAMP detection system was constructed based on the extracted DNA as the detection template, and its performance was evaluated. We present the following article in accordance with the STARD reporting checklist (available at https://dx.doi.org/10.21037/apm-21-1387).

\section{Methods}

\section{Research objects}

\section{Experimental strains}

The clinical strains, Burkbolderia cepacia, Klebsiella pneumoniae, Enterococcus faecium, Serratia marcescens, Staphylococcus epidermidis, Methicillin-resistant Staphylococcus aureus, Citrobacter freundii, Strange Proteus, Salmonella enterica, Brucella, Carbapenam-resistant Klebsiella pneumoniae (CRKP), Carbapenam-resistant A. baumannii (CRAB), carbapenem-resistant Pseudomonas aeruginosa (CRPA), Carbapenem-resistant enterobacteriaceae (CRE) were used in this study.

The standard strains, Shigella ATCC25931, Acinetobacter baumannii ATCC170, Enterobacter cloacae ATCC700323, Enterococcus faecalis ATCC29212, Pseudomonas aeruginosa ATCC27853, Haemophilus influenzae ATCC9007, Stenotrophomonas maltophilia ATCC17666, Staphylococcus aureus ATCC29213, Proteus vulgaris ATCC13315, and Escherichia coli ATCC25922, were also obtained.

According to the data from China Antimicrobial Resistance Surveillance System (CHINET), the strain types covering more than $95 \%$ of the clinical departments of hospital were included. In addition, a good resuscitation culture activity of the strain, as confirmed by a microbial mass spectrometer, was also required. Strains that did not comply with the above conditions were excluded.

Resuscitation of experimental strains was performed as follows: A paper sheet dipped in the strain was gently plated onto one side of a blood plate by sterile tweezers. This plate was considered the first zone. The other two zones were delineated by sterilized inoculation rings and incubated in incubator at $37^{\circ} \mathrm{C}$ for 24 hours for bacteria enrichment. The growth and activity of colony were observed and confirmed by the VITEK MS microbial identification mass spectrometer (bioMérieux SA, Marcy L'Etoile, France).

\section{Instruments and reagents}

As stated above, a VITEK MS microbial identification mass spectrometer was acquired from bioMérieux SA. Other devices and materials obtained for the experiment were the following: a SLAN-96P real-time fluorescent quantitative PCR instrument (Shanghai Hongshi, Shanghai, China), a Nano-400A micro spectrophotometer (Hangzhou Allshen, Hangzhou, China), a KZ-11 high-speed tissue grinder (Servicebio, Wuhan, China), a metal bath (KylinBell, Haimen Qilin, Jiangsu, China), a LAMP fluorescence detection kit and DNA-LAMP amplification reaction kit (Beijing Lanpu, Beijing, China), a Bacterial Genomic DNA Extraction Kit and lysozyme (Tiangen Biotech, Beijing, China), and a freeze-dried LAMP amplification kit (HNB color change; Beijing Anxinkang, Beijing, China). The primers were synthesized by Sangon Biotech (Beijing, China). 


\section{Experimental methods}

\section{Design and synthesis of primers}

Salmonella strains and nucleic acid sequences of multiple target fragments were searched for in the Nucleotide database of the National Center for Biotechnology Information (NCBI). The conserved regions were searched in the sequence alignment software, and those conserved regions of target sequences less than 2,000 bp were selected for the design template. After logging into LAMP online primer design website (http:// primerexplorer.jp/lampv5e/index.html), we uploaded the target conservative sequence for primer design, for which sequence alignment was performed in the basic local alignment search tool (BLAST) functional area of NCBI, and the specificity and conservation of the primers were evaluated. After the primers were designed and synthesized, the best primers were screened and selected among the many primers.

\section{DNA extraction of the bacterial genome}

The extraction of strains and sample DNA were performed in accordance with the instructions of the Bacterial and Fecal Genomic DNA Extraction Kit, respectively. After extraction, the purity and concentration of DNA were detected with a microspectrophotometer.

\section{DNA-LAMP detection reaction}

The total reaction volume of detection system was $25 \mu \mathrm{L}$ according to fluorescence and visual observation. The FAM (Carboxyfluorescein) channel was selected for fluorescence detection, and the reaction was conducted at $63{ }^{\circ} \mathrm{C}$ for 45 minutes. The fluorescence signal was collected every 30 or 60 seconds, and the reaction was terminated at $80^{\circ} \mathrm{C}$ for 10 minutes. The color change was observed immediately by a visual observation detection system after 30 minutes of reaction (Table 1).

\section{Evaluation of the results}

The positive amplification curve of the fluorescence detection system was shaped as "S". If the cycle threshold $(\mathrm{Ct})$ value was $<30$ minutes, it was considered positive; While when the $\mathrm{Ct}$ value was $>35$ minutes, it was considered negative. It should be reverified if the $\mathrm{Ct}$ value was between 30 and 35 minutes. Evaluation by visual observation was performed according to color change. A color change from violet to sky blue was considered positive, while no color change was considered negative.

\section{Testing of the simulated sample}

Next, 180-220 mg of 30 randomly selected stool specimens with a negative result after bacterial culture were placed into a $2-\mathrm{mL}$ centrifuge tube. The bacterial culture was mixed with $1 \mathrm{~mL}$ of sterilized $\mathrm{ddH}_{2} \mathrm{O}$ water, with a bacterial concentration of approximately $3 \times 10^{8} \mathrm{cfu} / \mathrm{mL}$ being estimated by the McFarland turbidimetric method. The bacterial culture was centrifuged then the supernatant was removed. The precipitation was mixed with the fecal sample, and placed into the high-speed tissue grinder. The genomic DNA was extracted according to the Genomic DNA extraction reagent box instructions and amplified by LAMP, and the results were observed.

\section{Statistical methods}

In this study, descriptive research was used to organize the experimental data into a four grid table format, and the calculation was carried out according to the following formula.

\begin{tabular}{lcccc}
\hline & \multicolumn{2}{c}{ Comparison method } & \multirow{2}{*}{ Total } \\
\cline { 3 - 4 } & Positive & Negative & \\
\hline Experimental & Positive & $\mathrm{a}$ & $\mathrm{b}$ & $\mathrm{a}+\mathrm{b}$ \\
methods & Negative & $\mathrm{c}$ & $\mathrm{d}$ & $\mathrm{c}+\mathrm{d}$ \\
& & $\mathrm{a}+\mathrm{c}$ & $\mathrm{b}+\mathrm{d}$ & $\mathrm{n}$ \\
\hline
\end{tabular}

Total coincidence rate $=100 \% \times[(\mathrm{a}+\mathrm{d}) / \mathrm{n}]$; Positive coincidence rate $=100 \% \times[a /(a+b+c)] ;$ Negative coincidence rate $=100 \% \times$ $[d /(c+d+b)] ;$ Sensitivity $=a /(a+b) ;$ Specificity $=d /(c+d)$

\section{Results}

\section{Screening of LAMP primers}

Primers were designed and screened for the inv $A$ gene of Salmonella, and amplified by the LAMP method; the positive reaction was indicated by a typical S-shaped curve and specific color changes, while a negative result was indicated by no S-shaped curve or color change. The primer sequences which can give the positive results were selected and shown in Table 2.

\section{Effects of different reaction temperature, primer amount and reaction time on the results}

After the primers were screened, different amounts of the primers $(1,1.5$ and $2 \mu \mathrm{L})$ were added and cultured for 
reaction time $(20,25,30$ and 45 minutes $)$ at a reaction temperature of $60-65^{\circ} \mathrm{C}$, and the differences between the results were observed. The results showed that different reaction temperatures and different primer loading volumes had no obvious effect on the results, and the negative and positive results could be clearly judged within 25 and 30 minutes of reaction time (Figure $1 A, B, C$ ).

Table 1 LAMP reaction system of Salmonella

\begin{tabular}{lc}
\hline Reagent & Volume $(\mu \mathrm{L})$ \\
\hline $\begin{array}{l}\text { Mix primers (including inner, outer and loop } \\
\text { primers) }\end{array}$ & 2 \\
Buffer Mix & 21 \\
Bacterial DNA & 1 \\
DNA polymerase & 1 \\
Total volume & 25 \\
\hline
\end{tabular}

Table 2 The best primer sequence

\begin{tabular}{ll}
\hline Primer name & Primer sequence \\
\hline F3 & GAACGTGTCGCGGAAGTC \\
B3 & CCACCGAAATACCGCCAAT \\
FIP & GCGCGGCATCCGCATCAATAATGGTATGCCCGG \\
& TAAACAG \\
BIP & AGGGAAAGCCAGCTTTACGGTTTAATGATGCCG \\
& GCAATAGCG \\
LF & CCTTCAAATCGGCATCAATACTCAT \\
LB & CCTTTGACGGTGCGATGAAGTTAT \\
\hline
\end{tabular}

\section{Sensitivity of the method}

The concentration and purity of extracted Salmonella DNA was tested. The DNA was 10 -fold diluted with sterile $\mathrm{ddH}_{2} \mathrm{O}$ and tested after LAMP reaction. The lowest detectable concentration of DNA was considered to indicate the sensitivity of the system. The fluorescence detection system was diluted into $\mathrm{ddH}_{2} \mathrm{O}$ at $10^{2}, 10^{3}, 10^{4}, 10^{5}, 10^{6}$ and $10^{7}$ fold, and the related Ct values were 20.99, 24.85, 33.31, $37.96,43.99$, and 41.46 respectively, yielding a sensitivity of $1.4 \mathrm{pg} / \mu \mathrm{L}$. When the system was diluted at $10^{2}, 10^{3}$, $10^{4}, 10^{5}, 10^{6}$, and $10^{7}$ in solution for visual observation, the sensitivity was $1.4 \mathrm{pg} / \mu \mathrm{L}$ (Figure 2).

\section{Specificity of method}

The extracted bacteria identified were detected by mass spectrometry to verify the specificity of the LAMP method. The Ct value of Salmonella in the fluorescence detection system was 21.07 , with the rest species of bacteria being negative with $100 \%$ specificity (Table 3 and Figure 3 A). Only Salmonella was positive in visual observation, while the rest were negative, yielding a $100 \%$ specificity (Figure 3B,C,D,E,F).

\section{Coincidence rate of evaluation methods with contaminated samples and clinical samples}

The DNA from the detection of 30 fecal specimens contaminated with Salmonella and 30 fecal specimens without Salmonella contamination was extracted according to the methods and procedures described above, and tested
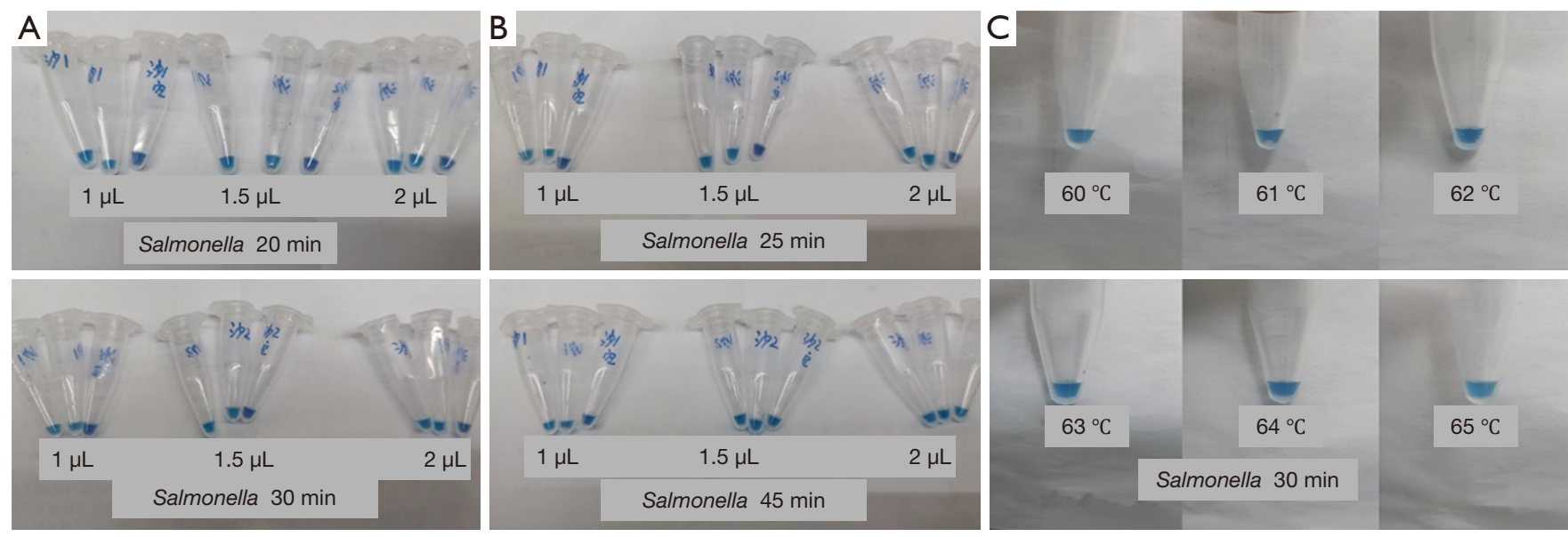

Figure 1 Color changes of Salmonella at different reaction temperatures, amounts of primer, and reaction times. 

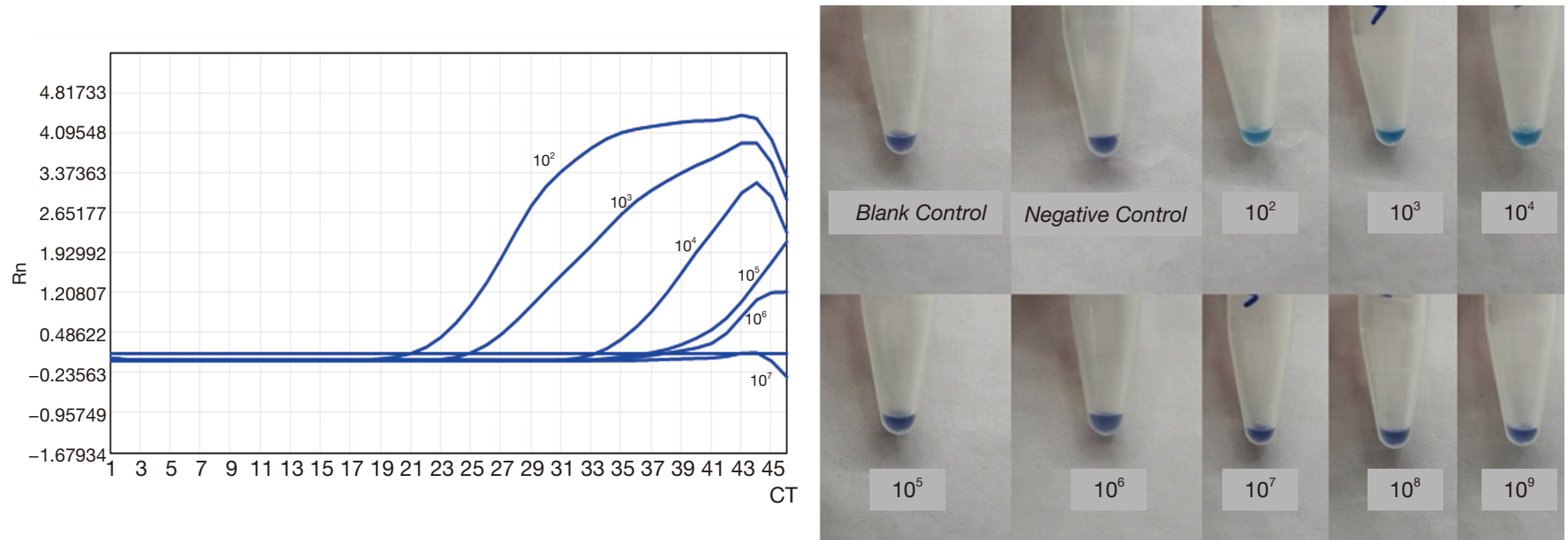

Figure 2 Sensitivity results of the LAMP method for Salmonella. LAMP, loop-mediated isothermal amplification.

Table 3 Specificity results for Salmonella detection

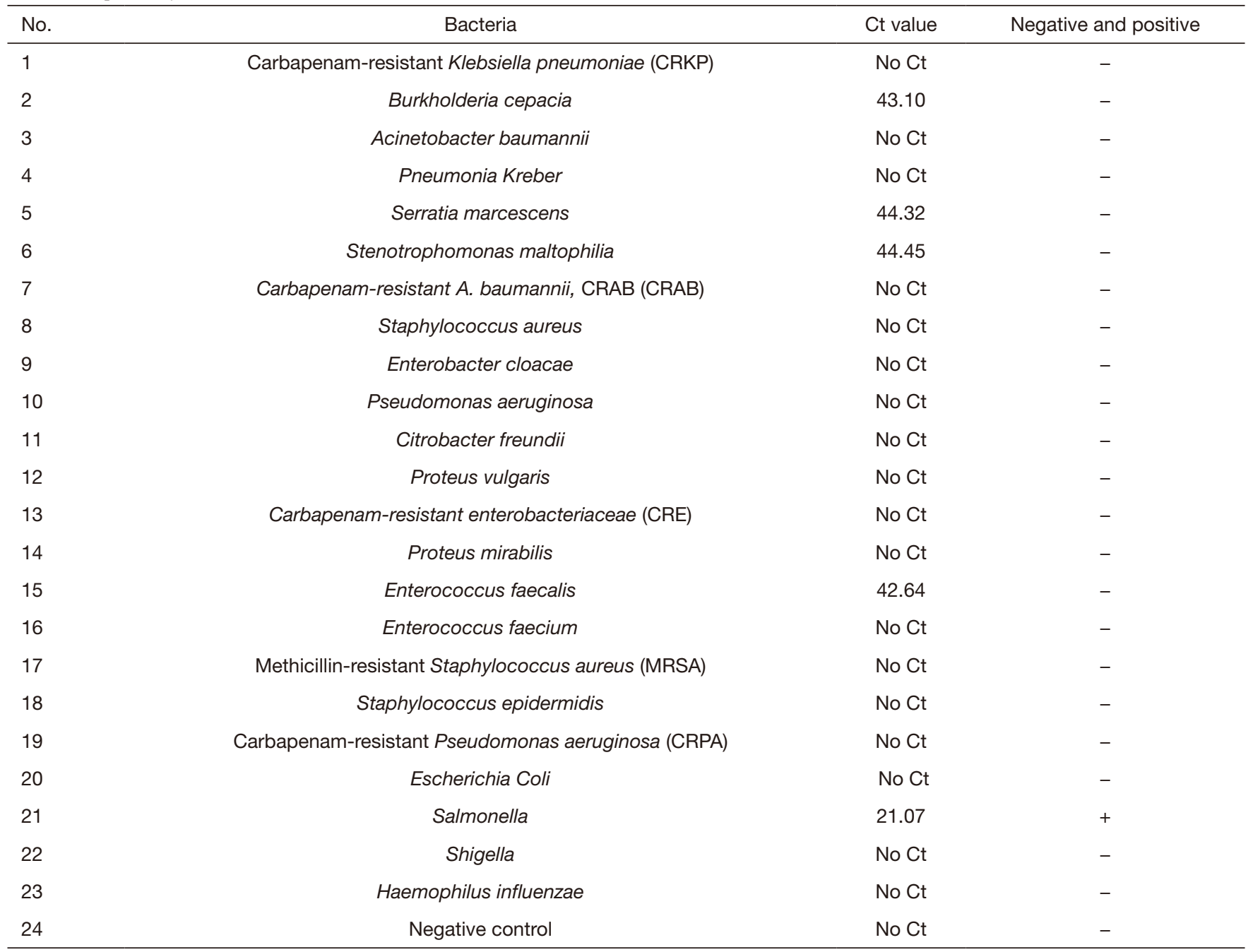

Ct, cyclic threshold. 
A
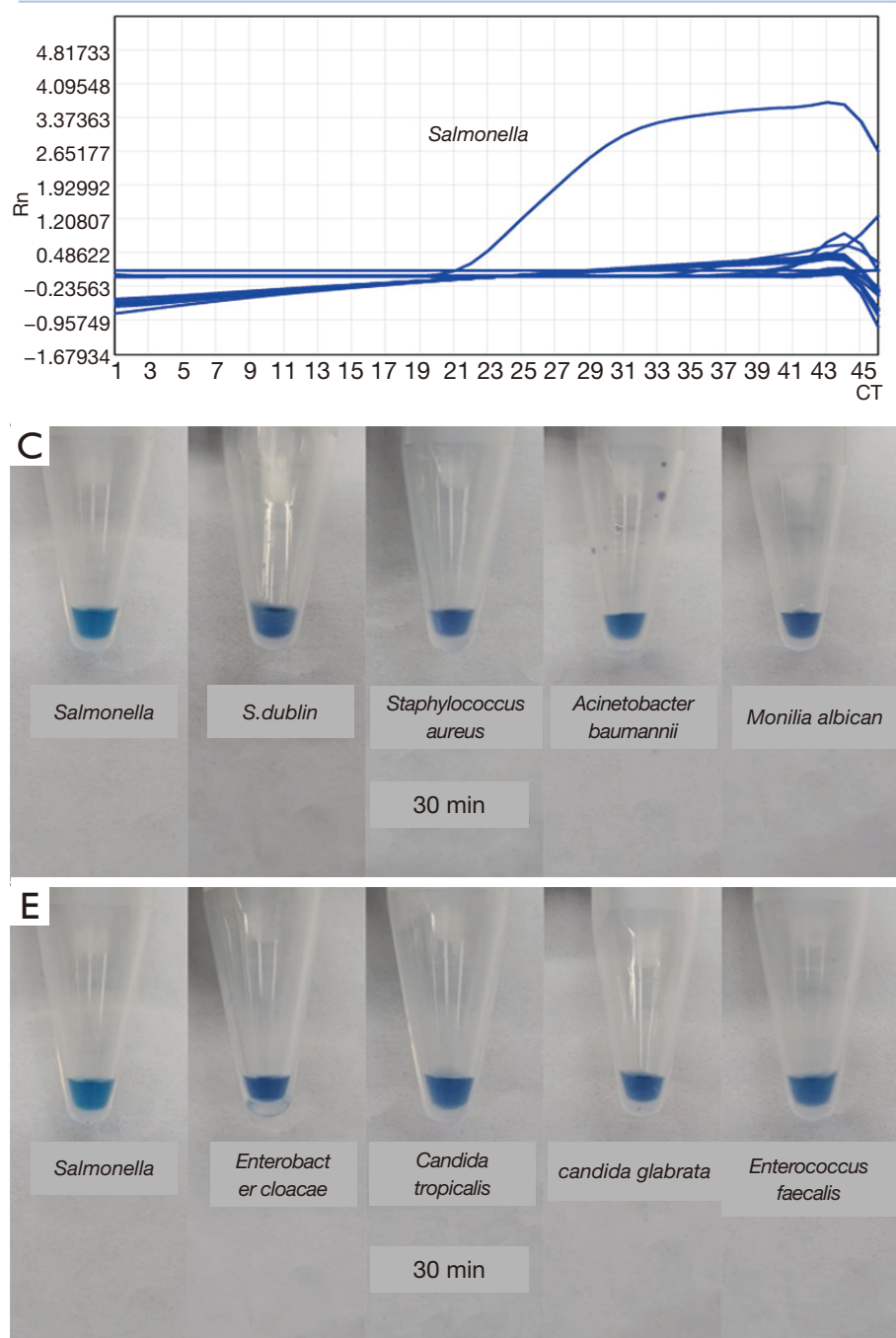

B
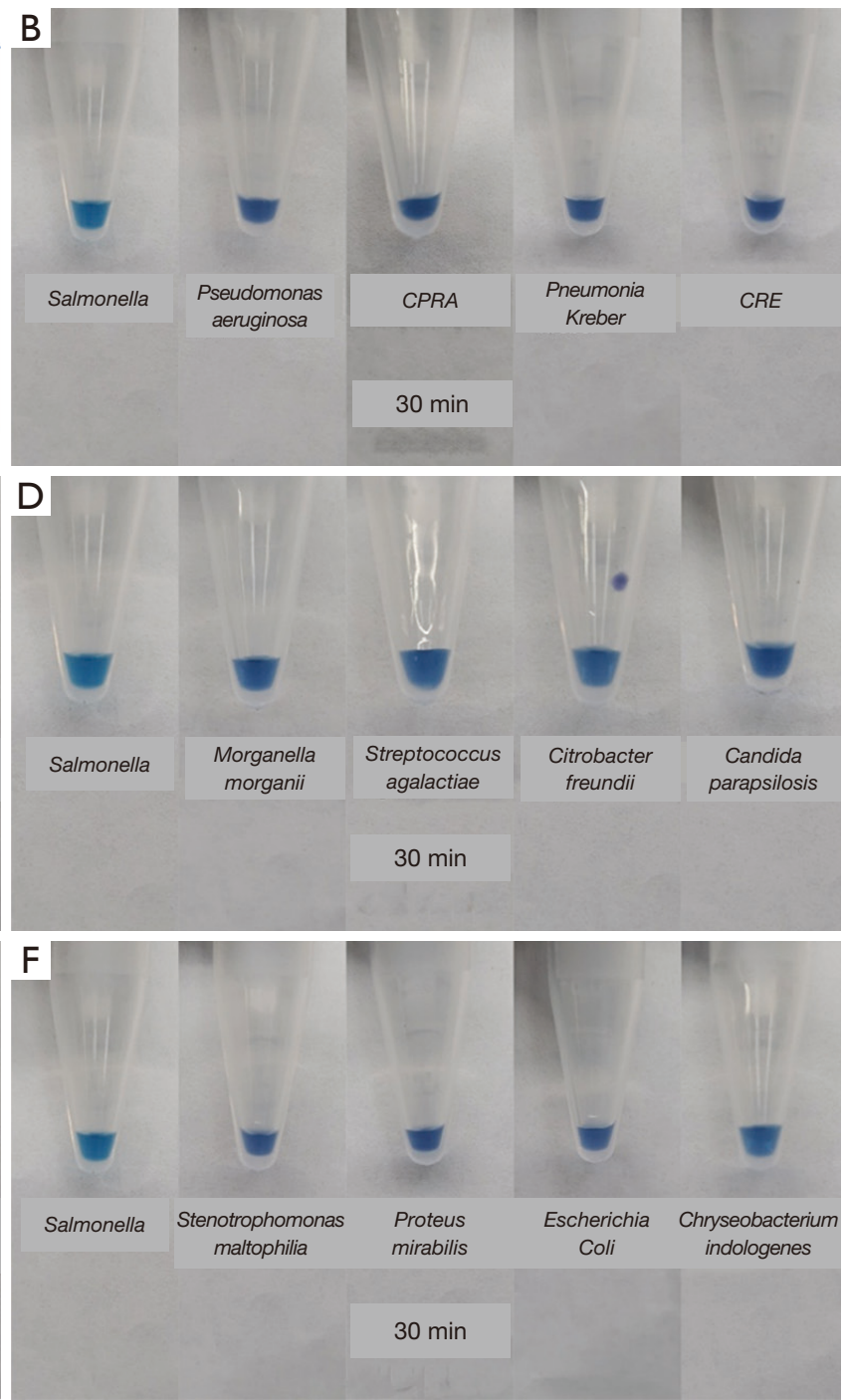

Figure 3 Specificity results of the LAMP method for Salmonella. LAMP, loop-mediated isothermal amplification.

by the 2 detection systems. In all, 29 simulated positive specimens were identified as positive by the fluorescence detection system, and 27 simulated positive specimens were identified as positive by the visual observation system, while 30 negative specimens were identified as negative. In 8 clinical samples identified as positive for Salmonella by bacterial culture, 8 samples were identified as positive by the fluorescence detection system, 7 samples and 1 sample were identified as positive and negative by the visual observation, respectively (Table 4). The positive coincidence rates of the real-time fluorescence and visual observation were $97.4 \%$ and $89.5 \%$, respectively, the negative coincidence rates were both $100 \%$, and the total coincidence rates were $98.5 \%$ and $94.1 \%$, respectively. The composition of fecal sample is complex, with many interference factors being present that can easily influence test results. However, the results indicated that the 2 LAMP-based detection methods established by the research institute demonstrated good coincidence and good anti-interference ability, and could be used to directly test the specimens.

\section{Discussion}

Salmonellosis, also known as paratyphoid, is a general term for infectious diseases of animals and humans infected by Salmonella spp. Salmonella is an important zoonotic pathogen that causes foodborne diseases in public health (World Health Organization, 2017) with many reports 
Table 4 Test results of contaminated samples, uncontaminated samples, and clinical samples

\begin{tabular}{|c|c|c|c|c|c|c|}
\hline Sample & Type of sample & Cases & \multicolumn{2}{|c|}{ Fluorescence } & \multicolumn{2}{|c|}{ Visual observation } \\
\hline Contaminated sample & Feces & 30 & 29 & 31 & 27 & 33 \\
\hline Uncontaminated sample & Feces & 30 & 0 & 30 & 0 & 30 \\
\hline Clinically positive samples & Blood, urine and feces & 8 & 8 & 0 & 7 & 1 \\
\hline
\end{tabular}

describing its lethality in infected individuals (9-11). There is a wide array of Salmonella hosts, and Salmonella can spread with ease, directly or indirectly in nature. There are two main types of salmonellosis infection in humans: typhoid and nontyphoid salmonellosis. Typhoid is an acute intestinal disease characterized by high fever, diarrhea, and leukopenia. In severe cases, it may even be complicated by intestinal perforation.

In the prevention and treatment of infectious diseases, the traditional bacterial culture method is the gold standard for detecting pathogenic bacteria in infectious diseases, but requires a high level of professional expertise, a variety of instruments and reagents, and a long detection time. Therefore, the bacterial culture method only can be used in large medical institutions, with timely diagnosis by bacterial culture in primary medical institutions and incident scenes being impracticable. The traditional bacterial rapid test method depends mainly on the use of unique biochemical reactions and selective media for bacterial identification. It relies on a culture method that requires over 12 hours to complete and that can only test for a few bacterial species. Another bacterial rapid test, the colloidal gold method, has simple operation and short reaction time, but it is prone to false positives or false negatives and has low sensitivity and specificity. To compensate for these shortcoming, research into the rapid detection of pathogenic bacteria using molecular biology techniques (such as PCR, gene chip, strand replacement technologies, autonomous replicating sequences, and others) has made great progress (12-19); nonetheless, modern detection still requires expensive nucleic acid amplification equipment, highly trained personnel, complex procedures, and a detection time of 3-5 hours. Given these restrictions, it can only be performed in large medical institutions, and its application as a rapid diagnostic method is also limited. When faced with the extreme conditions, such as those found in frontline medical institutions, intestinal infectious disease outbreaks, disaster areas, incident scenes of public health infection, etc., a rapid identification of microorganisms is required. This is made more difficult given the limitations of environment and equipment and the need for improved detection accuracy.

LAMP is a new molecular biology method that was first innovated by Notomi et al. in 2000. In this method, 4 specific primers are designed for 6 regions of the target gene. Nucleic acid amplification is performed by stranddisplacing DNA polymerase at constant temperature conditions. The results are evaluated based on the change in fluorescence intensity or color by changing the turbidity of the magnesium pyrophosphate precipitation or adding dyes. The whole reaction time lasts only 30 minutes to 1 hour. The technical requirements are simple and relatively cost-effective, and can be satisfied by any equipment with a stable heat source or an ordinary water bath (20-22).

In this study, a rapid detection method for Salmonella based on LAMP technology was established. Quality control was performed on several aspects of the primer design, and the key factors of the primer design were determined through exploration. Good primers need appropriate Tm (melting temperature) value and GC (guanine cytosine) pair content, and good stability of primer end and the interdistance of primers. These could help to avoid secondary structures. In the experiment, the conserved region with the target inv $A$ gene sequence was selected as template to design primers, and the primers were sequenced in the BLAST functional region to fully evaluate the specificity and conservation of the primers to be screened. By controlling the key factors of primer design, the best primers were obtained to guarantee the good performance of the detection system. After the screening of the best primers, the reaction conditions were further optimized to observe the accuracy of the detection system for the standard strains at variable temperatures, primer amounts, and reaction times. When the reaction temperature was between $60-65{ }^{\circ} \mathrm{C}$ and the amount of primer was $1-2 \mu \mathrm{L}$, the results showed no significant difference. The results were stable within 25-30 minutes of reaction. The extracted bacterial DNA was tested 
after multiple dilution. The detection sensitivity of the fluorescence detection system was $1.4 \mathrm{pg} / \mu \mathrm{L}$, and the detection sensitivity of the visual observation detection system was $1.4 \mathrm{pg} / \mu \mathrm{L}$. The specificity of the 2 detection systems was $100 \%$. In 30 positive specimens contaminated with Salmonella, 29 specimens and 27 specimens were identified as positive by fluorescence and visual observation detection system, respectively, and 30 negative specimens were identified as negative; all positives showed typical S-curve and positive color changes. Eight clinical samples that were cultured as positive for Salmonella were detected, 8 specimens were positive by the fluorescence detection system, 7 specimens were identified as positive by the visual observation, and 1 specimens was identified as negative. The positive coincidence rate, negative coincidence rate, and total coincidence rate of fluorescence and visual observation detection system were $97.4 \%$ and $89.5 \% ; 100 \%$ and $100 \%$; and $98.5 \%$ and $94.1 \%$, respectively. The false-negative results of 2 contaminated samples and 1 clinical sample in visual observation was analyzed: 2 cases showed no obvious color change, and 1 case was negative. This discrepancy might have been caused by differences during visual observation. It also indicates that the accuracy of visual observation system is slightly lower than that of the fluorescence detection system. Another test case was identified as negative in the 2 systems possibly due to DNA loss from improper operation during sample processing and bacterial DNA extraction. Due to the complex composition of fecal samples and many interference factors, the sample size of this study was limited, and further research should be conducted with a lager sample size and a greater variety of sample types.

Our study demonstrated that combining LAMP technology with fluorescent and dye indicators could provide a method for real-time monitoring of the entire constant temperature amplification process. The results could be intuitively evaluated by fluorescence curve and visual observation after completion of the LAMP reaction over 25-30 minutes. With the addition of fluorescence and dye in advance, both methods can prevent the formation of aerosols and avoid the false-positive results caused by environmental pollution, without the need for opening the lid and adding result indicators or applying electrophoresis. In conclusion, the LAMP-based technology with the inv $A$ target gene established in this study can accurately detect Salmonella without expensive detection instruments. It represents a new means of rapid screening detection for
Salmonella, particularly in the high-demand, resourcelimited conditions of primary laboratories, disaster treatment areas, and infection sites, among others.

\section{Acknowledgments}

Funding: Youth training program of military medical science and technology (16QNP030).

\section{Footnote}

Reporting Checklist: The authors have completed the STARD reporting checklist. Available at https://dx.doi. org/10.21037/apm-21-1387

Data Sharing Statement: Available at https://dx.doi. org/10.21037/apm-21-1387

Conflicts of Interest: All authors have completed the ICMJE uniform disclosure form (available at https://dx.doi. org/10.21037/apm-21-1387). The authors have no conflicts of interest to declare.

Etbical Statement: The authors are accountable for all aspects of the work in ensuring that questions related to the accuracy or integrity of any part of the work are appropriately investigated and resolved.

Open Access Statement: This is an Open Access article distributed in accordance with the Creative Commons Attribution-NonCommercial-NoDerivs 4.0 International License (CC BY-NC-ND 4.0), which permits the noncommercial replication and distribution of the article with the strict proviso that no changes or edits are made and the original work is properly cited (including links to both the formal publication through the relevant DOI and the license). See: https://creativecommons.org/licenses/by-nc-nd/4.0/.

\section{References}

1. Knodler LA, Elfenbein JR. Salmonella enterica. Trends Microbiol 2019;27:964-65.

2. Ledlod S, Bunroddith K, Areekit S, et al. Development of a duplex lateral flow dipstick test for the detection and differentiation of Listeria spp. and Listeria monocytogenes in meat products based on loop-mediated isothermal amplification. J Chromatogr B Analyt Technol Biomed Life Sci 2020;1139:121834. 
3. Wachiralurpan S, Sriyapai T, et al. Development of a Rapid Screening Test for Listeria monocytogenes in Raw Chicken Meat Using Loop-Mediated Isothermal Amplification (LAMP) and Lateral Flow Dipstick (LFD). Food Analytical Methods. 2017.

4. Birmpa A, Kouroupis G. A Loop-mediated Isothermal Amplification Platform for the Detection of Foodborne Pathogens. Bioeng. Biomed 2015.

5. Meng X, Zhang G, Sun B, et al. Rapid Detection of mecA and femA Genes by Loop-Mediated Isothermal Amplification in a Microfluidic System for Discrimination of Different Staphylococcal Species and Prediction of Methicillin Resistance. Front Microbiol 2020;11:1487.

6. Nguyen AV, Orlofsky A, Pubill K, et al. Loop-mediated isothermal amplification (LAMP) as a rapid, affordable and effective tool to involve students in undergraduate research. Front Microbiol 2020;11:603381.

7. Liu Z, Zhang Q, Yang NN, et al. Rapid and Sensitive Detection of Salmonella in Chickens Using Loop-Mediated Isothermal Amplification Combined with a Lateral Flow Dipstick. J Microbiol Biotechnol 2019;29:454-64.

8. Mashooq M, Kumar D, Niranjan AK, et al. Development and evaluation of probe based real time loop mediated isothermal amplification for Salmonella: A new tool for DNA quantification. J Microbiol Methods 2016;126:24-9.

9. Ma Y, Li M, Xu X, et al. High-levels of resistance to quinolone and cephalosporin antibiotics in MDR-ACSSuT Salmonella enterica serovar Enteritidis mainly isolated from patients and foods in Shanghai, China. Int J Food Microbiol 2018;286:190-6.

10. Almanza AV. Interagency Food Safety Analytics Collaboration (IFSAC): Meeting on Work to Improve Foodborne Illness Source Attribution Estimates for Salmonella, Escherichia coli O157, Listeria, and Campylobacter. 2015.

11. Davidson VJ, Ravel A, Nguyen TN, et al. Food-specific attribution of selected gastrointestinal illnesses: estimates from a Canadian expert elicitation survey. Foodborne Pathogens \& Disease 2011;8:983-95.

12. Wen J, Gou $\mathrm{H}$, Zhan Z, et al. A rapid novel visualized loop-mediated isothermal amplification method for Salmonella detection targeting at fimW gene. Poult Sci

Cite this article as: Ou H, Wang Y, Gao J, Bai J, Zhang Q, Shi L, Wang X, Wang C. Rapid detection of Salmonella based on loop-mediated isothermal amplification. Ann Palliat Med 2021;10(6):6850-6858. doi: 10.21037/apm-21-1387
2020;99:3637-42.

13. Abirami N, Nidaullah H, Chuah LO, et al. Evaluation of commercial loop-mediated isothermal amplification based kit and ready-to-use plating system for detection of Salmonella in naturally contaminated poultry and their processing environment. Food Control 2016;70:74-8.

14. Hu L, Ma LM, Zheng S, et al. Evaluation of 3M Molecular Detection System and ANSR Pathogen Detection System for rapid detection of Salmonella from egg products. Poult Sci 2017;96:1410-8.

15. Hu L, Ma LM, Zheng S, et al. Development of a novel loop-mediated isothermal amplification (LAMP) assay for the detection of Salmonella ser. Enteritidis from egg products. Food Control 2018;88:190-7.

16. Cheung PY, Chan CW, Wong W, et al. Evaluation of two real-time polymerase chain reaction pathogen detection kits for Salmonella spp. in food. Lett Appl Microbiol 2004;39:509-15.

17. Papadakis G, Murasova P, Hamiot A, et al. Micro-nano-bio acoustic system for the detection of foodborne pathogens in real samples. Biosens Bioelectron 2018;111:52-8.

18. Park BH, Oh SJ, Jung JH, et al. An integrated rotary microfluidic system with DNA extraction, loop-mediated isothermal amplification, and lateral flow strip based detection for point-of-care pathogen diagnostics. Biosens Bioelectron 2017;91:334-40.

19. Heymans R, Vila A, van Heerwaarden CAM, et al. Rapid detection and differentiation of Salmonella species, Salmonella Typhimurium and Salmonella Enteritidis by multiplex quantitative PCR. PLoS One 2018;13:e0206316.

20. Notomi T, Okayama H, Masubuchi H, et al. Loopmediated isothermal amplification of DNA. Nucleic Acids Res 2000;28:E63.

21. Martinović T, Andjelković U, Gajdošik MŠ, et al. Foodborne pathogens and their toxins. J Proteomics 2016;147:226-35.

22. Knowles M, Stinson S, Lambert D, et al. Genomic Tools for Customized Recovery and Detection of Foodborne Shiga Toxigenic Escherichia coli. J Food Prot 2016;79:2066-77.

(English Language Editor: J. Gray) 